\title{
DIMENSIONING ELECTRIC CHARGING POSTS FOR PEV
}

\author{
J.-I. CAIRO \\ IREC - Spain \\ icairo@irec.cat
}

\author{
Miquel CRUZ \\ IREC - Spain \\ mcruz@irec.cat
}

\author{
Andreas SUMPER \\ IREC and CITCEA-UPC Spain \\ asumper@irec.cat \\ sumper@citcea.upc.edu
}

\begin{abstract}
This paper represents a new analysis method for the estimation of the required charging infrastructure for the electric vehicles (EV'S). The study is based on a real metropolitan area, taking real data from transport habits, and a prediction of certain degree of penetration of EV's. To cope with this analysis, is the application of powerful tools, used for example in telecommunication systems such as Markov chains, and applied here into $E V$ 's necessities and charging stations infrastructures. This theory is applied in a real example to provide more insight on how can be applied in real circumstances.
\end{abstract}

\section{INTRODUCTION}

For many years, power grid facilities have not changed their configuration because the energy distribution concept has not provide many changes from important generation units of energy until power consumers. With the introduction of distributed energy resources (DER), this concept may change, and so will do distribution and transportation network, changing from a total radial topology into a mesh topology. In that sense, the introduction of $\mathrm{EV}$, is expected to influence demanding side and communication for the control and managing of the dispersed grid units.

With the EV's there will be new demand patterns, and distribution networks, with their sub-stations and the $\mathrm{LV}$ power transformers that are dimensioned to deliver electricity depending on the number of costumers in a given area, with the large penetration of EV's would result in potential saturation or overloading problems for the power grid as has been studied and reported [1]. The innovative approach that is being applied here, consist in the dimensioning of Charging stations (CS) due to certain demand of energy for EV's and also the analysis of congestion of such CS's and time of use or waiting time.

This study has been carried out based on actual data from a city of Spain (Vitoria) so we introduce an example with data values, obtained from a real scenario. The goal of this paper is not to introduce in deep detail this scenario but to present the procedure for obtaining the estimations. Based on this data we propose some hypothesis and assumptions that focuses the scope of possibilities without eliminating the thoroughness of the methodology.

\section{REQUIREMENTS OF EV'S}

In the analysis presented in this paper, it is firstly introduced some data that relates the necessities of EV's in terms of its energy consumption and, in other terms introduces some statistics on the number of vehicles that may circulate in an specific area and are prone to request energy from the power grid.

\section{Descripcion of observed area}

Data for this case scenario has been obtained from a $13.2 \mathrm{kV}$ network in the Spanish electric system, in the city of Vitoria (Álava) powered by two power transformers. The conventional demand in that region is of $36 \mathrm{MW}$ distributed in different areas as described in table I.

The assumptions for the EV penetration are taken from a study in the same project context, with the aim of estimating the power requested for charging EV's in the 2010-2020 horizon. From the different scenarios here described, for this study we have just considered the case of residential areas. Energy consumption is well known as well as habits from the habitants regarding mobility. We are using the assumptions of conventional combustion cars mobility data since this is the one available nowadays, in order to extrapolate towards mobility with EV's as described in the following paragraph.

TABLE I

DISTRIBUTION OF ENERGY DEMANDED IN THE METROPOLITAN AREA OF ALAVA (SPAIN) BY SECTORS

\begin{tabular}{lllll}
\hline \hline $\begin{array}{l}\text { Type of } \\
\text { Area }\end{array}$ & Residential & Commercial & Industrial & Others \\
\hline Load $(\%)$ & $13 \%$ & $7 \%$ & $68.4 \%$ & $11.6 \%$ \\
\hline
\end{tabular}

\section{Assumptions on mobility}

The other source of information provided for this study comes from the project MOVILIA2006 [5], which considers an average consumption for the EV of 200 $\mathrm{Wh} / \mathrm{km}$, a mean traveling distance of $15 \mathrm{~km}$, and the average speed for the electric vehicles is supposed to be $30 \mathrm{~km} / \mathrm{h}$, mainly inside urban areas. The capacity of the battery will depend on the type of vehicle, here we suppose an average EV with $25 \mathrm{kWh}$ of capacity. From these considerations the daily Request of Charge (RoC) will be in the order of $1 / 8$ of the battery capacity since $200 \mathrm{Wh} / \mathrm{km} \cdot 15 \mathrm{~km}=3 \mathrm{kWh}$. :

From the work [5], it is also obtained the number of commutes in auto and motorcycle either in a mean labor day as well as non-labor day in the province of Alava

- Number of Commutes in average labor day: 41.151.

- Number of Commutes in average non-labor day: 38.022 
And the number of commutes realized by the different type of $\mathrm{EV}$ is according to the following distribution:

- PHEV: $36.83 \%$

- $\mathrm{BEV}: 45.02 \%$

- Electric motorcycle: $18.15 \%$

In figure 1 one can see the number of commutes made by users of battery electric vehicles (BEV), due to people returning home in a labor day [2].

As a premise, we have chosen the time of the day during which the users will most probably use for recharging their EV's and that is during evening - night hours, and this is what is used for the next part of the study.

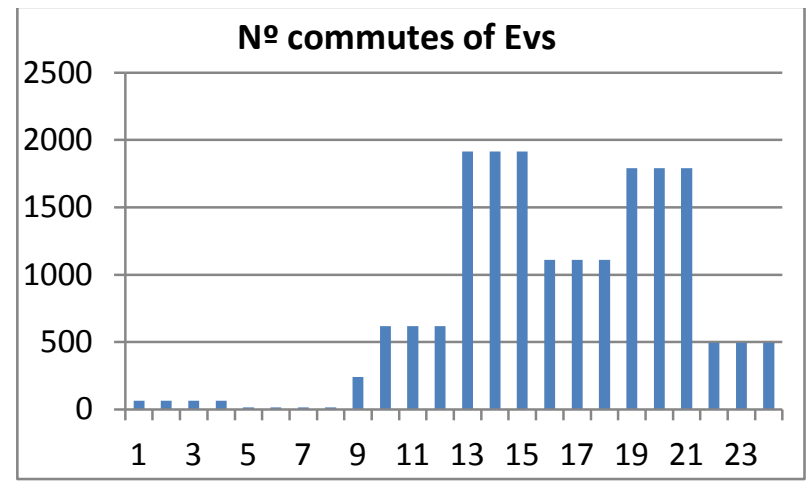

Figure 1. Number of commutes arring at user home during a whole labor day.

\section{Charging Request is a Poisson Distribution}

It is of importance to determine how the EV's arrive to the place of the CS and apply for a Request of Charge (RoC). Several scenarios can be introduced such as charging stations in the street areas, in public parking's, at home or in private parking from a community. Here implementation of CS's in a scenario where the EV users may have access to different charging points.

One of the most employed statistics to model such arrivals and RoC due to its simplicity and properties is the Poisson process. An important property of this distribution is of being a non memory system, since every arrival of the units is independent from when the previous arrival took place; infinite source, it makes it independent from the time window of observability to take average values. The probability of having an arrival into the system is proportional to the time $\Delta \mathrm{t}$, that's to say that $\lambda \cdot \Delta \mathrm{t}+\mathrm{O}(\Delta \mathrm{t})$, where $\mathrm{O}(\Delta \mathrm{t})$ is a Landau $\mathrm{O}$.

With previous hypothesis the probability of having $n$ arrivals of EV's at time $T$ (or $\Delta \mathrm{t}$ ) follows the following:

$$
P_{n}(t)=\frac{(\lambda T)^{n}}{n !} e^{-\lambda T}
$$

From data of figure 1, we take the assumption that our window time of interest is the one, as mention earlier, from 16 hours until 8 hours in the morning. In that window, we can obtain a distribution rate of $\lambda=4.2$, with $f_{i}$ as the relative frequency of observations:

$$
\lambda=\bar{X}=\frac{1}{n} \sum_{i=0}^{k} x_{i} \cdot f_{i} \quad f_{1}+f_{2}+\ldots+f_{k}=n
$$

In figure 2 is plotted the Poisson distribution of arrival probabilities comparing the measured data [2] and the estimated data.

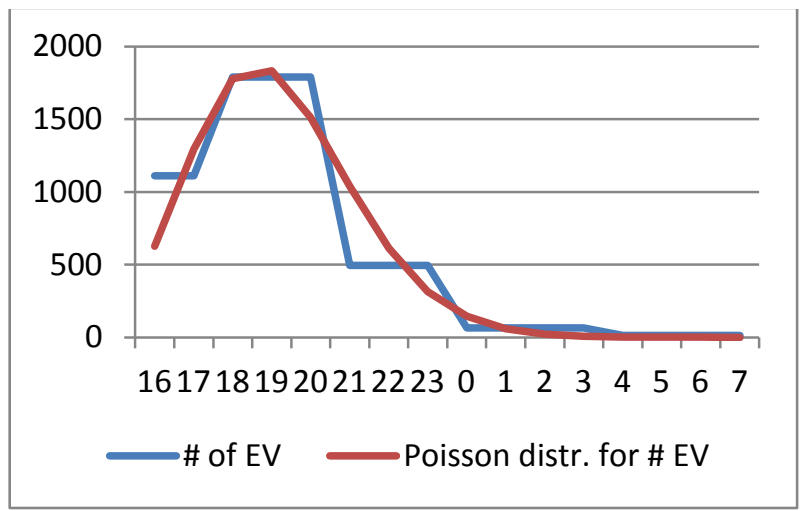

Figure 2. Poisson distribution of the number of home arrivals from $16 \mathrm{~h}$ until $7 \mathrm{~h}$.in a labor day.

\section{PROBABILITY FUNCTION DISTRIBUTION}

In the case under application, it is suggested a situation where a local manager (LM) is connected to a certain number of charging points $(\mathrm{CP})$, it is supposed a number of 4 CP's with certain capacity for charging batteries at slow speed. In the case of slow charging capabilities the maximum charging current is 16 Aac which means $3.7 \mathrm{~kW}$ of power at a $230 \mathrm{Vac}$ feeding line.

The power demanded by these charge stations are normally managed by LM in accordance to the maximum demand, energy price, incentives and power capabilities of the facilities. In the present case, the facility is accommodated with a three phase connection, where power can be obtained from any of the three phases independently until maximum demand is achieved. When an EV arrives, it demands for connectivity to the grid for a certain amount of energy, that is obtained from any of the phases, otherwise the EV waits until there is a phase available. The length of the energy charging request by the EV's arriving to the power station is suppose to be exponentially distributed with average value of $3 \mathrm{kWh}$ as will be further justified.

TABLE II

SYSTEMS USED FOR POSITIONING OF MOBILE TERMINALS

\begin{tabular}{lclcl}
\hline \hline $\begin{array}{l}\text { Average } \\
\text { consumption }\end{array}$ & $\begin{array}{c}\text { Average } \\
\text { travel dist. }\end{array}$ & $\begin{array}{l}\mathrm{N}^{\mathrm{o}} \\
\text { charging } \\
\text { stations }\end{array}$ & $\begin{array}{c}\text { Battery } \\
\text { capacity }\end{array}$ & $\begin{array}{l}\text { Average } \\
\text { RoC }\end{array}$ \\
\hline $200 \mathrm{Wh} / \mathrm{km}$ & $15 \mathrm{~km} /$ day $^{1}$ & 4 & $25 \mathrm{kWh}$ & $3 \mathrm{kWh}$
\end{tabular}

${ }^{1}$ This assumption considers residential use only. Other estimations $\mathrm{s}$ as work commuting, vacation or other would provide different value. 
The exponential distribution describes the time between events in a Poisson process, in which the events are succeeding continuously in time, independently and at an average constant speed. The probability distribution function follows:

$$
f(x)=\lambda \cdot e^{-\lambda x}
$$

Between the LM and the CP's there exists a protocol, so when an EV generates a message RoC, it remains inactive (does not generate any other message) until the $\mathrm{LM}$ validates that his request has been accepted. As soon as there is free energy available this energy request is serviced independently of any other parameter (price of energy, other local demands, etc). It is supposed that the arrival of EV's into the charging stations follows a Poisson distribution with a rate obtained from previous data of $\lambda=4.2 \mathrm{ev} / \mathrm{h}$, corresponding to the total observation period divided by 140 areas of the city.

\section{Block diagram of EV charging infrastructure}

In order to cover the $\mathrm{EV}$ energy requirements, there will be the need to dimension, locate and place several charging stations in public and private areas. During evening hours, when the users arrive home, they will request for charging energy for their vehicle. Using the Poisson distribution probability, we will try to compute the middle time an EV stays connected $(T)$ and the average number of EV's that stay connected into a charging station $(N)$.

In the following an assumption is made that there are four charging points providing energy. These four points are linked to a three phase power node that can obtain energy from the lines with a total contracted capacity of $10 \mathrm{~kW}$. If the lines are occupied the user will wait for availability of the node.

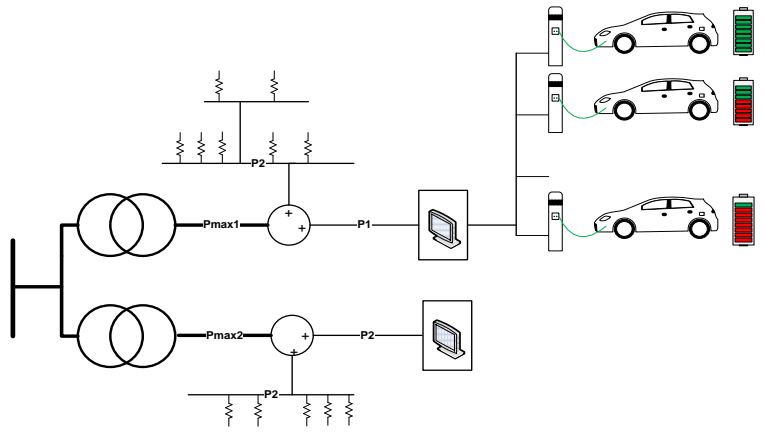

Figure 3. Percentage of commutes arring home for a labor day.

The topology depicted in figure 3, can be translated into an state space diagram as shown in figure 4 . This Markov diagram will allow us to study the probability variables of the system [3].

\section{Markov Process}

For the analysis of system traffic, in our case the traffic is defined as the energy flux through feeding lines, so after a RoC, the feeding line providing energy to the battery car, will be occupied during a determined period of time. To study this time of occupancy, it is used a well known tool, normally used for dimensioning telecommunication systems, based on the definition of certain states in which a system can be found, in order to find the probability of finding such system in one of those states. The theory of Markov processes, has also been applied to other fields, for example to analyze the failure rate on a transmission system, in that case also leads to a exponential probability distribution, corresponding to a system where more events are expected below the mean value of the random variables than above it, this probabilistic pattern is a classical model for electric systems reliability studies [4-5].

The probability that the system stays in the state $E m$ at the instant $t i$ is $\operatorname{Pr}[E m(t=t i)]$ or $\operatorname{Pm}(t i)$ and the stochastic vector state is:

$$
P\left(\left(t_{i}\right)=\left[P_{0}\left(t_{i}\right), P_{1}\left(t_{i}\right), P_{2}\left(t_{i}\right), \ldots\right]\right.
$$

From our previous introduction, our process will conceive 4 states each one associated with the probability of occupancy of one of these charging stations, so state 1 corresponds to the probability of finding the EV in the first charging point. Then we can define the Markov process as shown in figure 4 with four states of different transition rates, and the transition diagram, which defines the rate of entrance of new vehicles into the charging station, and also the rate of being serviced with the energy requested.

This type of diagram is restricted to the situation where from one state, only can change to the following neighbor state in the next time step, that is to say, from state $E_{n}$, one can only go to state $E_{n+1}$, to $E_{n-1}$ or stay at $E_{n}$. Such a way of proceeding defines what is called the birth and death processes. Such birth and death processes are characterized in steady state by the following equations:

$\begin{cases}0=\mu_{n+1} \cdot P_{n+1}+\lambda_{n-1} \cdot P_{n-1}-\left(\lambda_{n}+\mu_{n}\right) \cdot P_{n} & ; n>0 \\ 0=\mu_{1} \cdot P_{1}-\lambda_{0} \cdot P_{0} & ; n=0\end{cases}$

Where $\lambda$ is the stepping forward distribution rate and $\mu$ is the stepping backwards distribution rate. In this caser case $\lambda_{0}=4 \lambda, \lambda_{1}=3 \lambda_{2} \lambda_{2}=2 \lambda$, and $\lambda_{3}=\lambda$. Also $\mu_{1}=\mu$, $\mu_{2}=2 \mu, \mu 3=3 \mu$, and $\mu 4=3 \mu$.

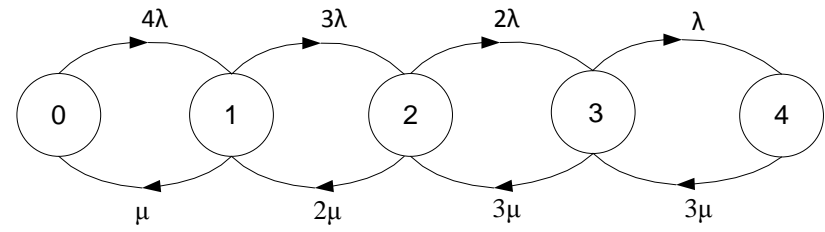

Figure 4. Diagram of the Markov process associated to the chargin stations probability of use. 


\section{Calculation of state probabilities from the process}

Previous equations can be solved and applied to this proposed example. Starting from the stat 0 and arriving to the last one, this is what one can get:

For $\mathrm{n}=0$;

$$
P_{1}=\frac{\lambda_{0}}{\mu_{1}} P_{0}
$$

For $\mathrm{n}=1$;

$$
\begin{gathered}
\mu_{2} \cdot P_{2}=P_{1} \cdot\left(\lambda_{1}+\mu_{1}\right)-\lambda_{0} \cdot P_{0}=\frac{\lambda_{0}}{\mu_{1}} P_{0} \cdot\left(\lambda_{1}+\mu_{1}\right)-\lambda_{0} \cdot P_{0} \\
\mu_{2} \cdot P_{2}=\frac{\lambda_{0} \cdot \lambda_{1}}{\mu_{1}} P_{0} \rightarrow P_{2}=\frac{\lambda_{0} \cdot \lambda_{1}}{\mu_{1} \cdot \mu_{2}} P_{0}
\end{gathered}
$$

Iterating for $\mathrm{n}=\mathrm{k}$ one can get:

$$
P_{k}=P_{0} \frac{\lambda_{0} \cdot \lambda_{1} \cdot \ldots \cdot \lambda_{k-1}}{\mu_{1} \cdot \mu_{2} \cdot \ldots \cdot \mu_{k}}=P_{0} \cdot \prod_{i=0}^{k=1} \frac{\lambda_{i}}{\mu_{i+1}}
$$

And the value of $P_{0}$ can be obtained from

$$
\sum_{k=0}^{\infty} P_{k}=1 ; \quad P_{0}=\frac{1}{1+\sum_{k=1}^{\infty} \prod_{i=0}^{k-1} \frac{\lambda_{i}}{\mu_{i+1}}}
$$

Lets solve it in our case. Before that, based on the capacity of the feeding line and the average RoC we will find the service rate:

$$
\mu=\frac{C}{R o C}=\frac{10 k W h}{3 k W h}=3,33
$$

So we can propose a set of equations that estimate the probability of finding the charging stations in any of the states:

$$
\begin{gathered}
p_{1}=4 \frac{\lambda}{\mu} \cdot p_{0}=15,12 \cdot p_{0} \\
p_{2}=6\left(\frac{\lambda}{\mu}\right)^{2} p_{0}=85,73 p_{0} \\
p_{3}=4\left(\frac{\lambda}{\mu}\right)^{3} p_{0}=216,04 p_{0} \\
p_{4}=\frac{4}{3}\left(\frac{\lambda}{\mu}\right)^{4} p_{0}=271,21 p_{0} \\
p_{0}(1+15.12+85.73+216.04+272.21)=1
\end{gathered}
$$

Solving for these equations we obtain that $p_{0}=0,0017$, $p_{1}=0,025, p_{2}=0,145, p_{3}=0,366$ and $p_{4}=0,461$. With this values one can easily compute the average number of $\mathrm{EV}$ in the area of charging stations;

$$
N=\sum_{k=0}^{4} k \cdot p_{k}=p_{0}\left[4\left(\frac{\lambda}{\mu}\right)+12\left(\frac{\lambda}{\mu}\right)^{2}+12\left(\frac{\lambda}{\mu}\right)^{3}+\frac{16}{3}\left(\frac{\lambda}{\mu}\right)^{4}\right]
$$

This is $\mathrm{N}=3,26$ so four EV's are in the charging location as average value during one hour. With this value, it is possible using the Little Formula, to compute the average time that an EV stays in the system $T=N / \lambda_{c}$, where $\lambda_{c}$, is the cumulative $\mathrm{RoC}$ rate:

$$
\begin{gathered}
\lambda_{c}=\sum_{k=0}^{3} \lambda_{k} \cdot p_{k}=4 \lambda p_{0}\left[1+3\left(\frac{\lambda}{\mu}\right)+3\left(\frac{\lambda}{\mu}\right)^{2}+\left(\frac{\lambda}{\mu}\right)^{3}\right] \\
\lambda_{c}=4 \lambda \cdot p_{0}\left(1+\frac{\lambda}{\mu}\right)^{3}=3,11
\end{gathered}
$$

So for the average time of an $\mathrm{eV}$ staying in this system with four charging station it corresponds to the value of $\mathrm{T}=60 \cdot 3,26 / 3,11=62,9 \mathrm{~min}$.

\section{CONCLUSIONS}

In this paper, we have presented a methodology for dimensioning the charging station using the Markov process and considering several assumption based on an scenario from Alava. By knowing the input EV entrance rate, and using the presented methodology one can forecast parameters such as average waiting time for the user and average number of users using the infrastructure.

One can expect such methodology to be applied in an emerging scenario such as the penetration of EV.

(11)Using this method one can forecast several parameters of interest.

\section{REFERENCES}

[1] J. Taylor, et al, 2009, "Evaluation of the impact of plug-in electric vehicle loading on distribution system opeations", IEEE Power and Energy Society General Meeting, Calgary, Canada, July.

[2] Project MOVILIA2006. Ministerio de Fomento.

[3] Sheldon M. Ross, 2007, "Introduction to Probability Models", $9^{\text {th }}$ edition, Elsevier.

[4] L.E. Aparicio, "Stochastic Reliability Study of the Western 765, 400 and $230 \mathrm{kV}$ transmission Lines Maintained by EDELCA", Yorozu, M. Hira

[5] R. Billinton and K. E. Bollinger, 1968 "Transmission System Reliability Evaluation Using Markov Processes,", IEEE Trans. PAS-87, No.2, Feb, pp 538-547.a 